\title{
MedienPädagogik
}

Zeitschrift für Theorie und Praxis der Medienbildung

Themenheft Nr. 38: Aneignung politischer Information in Social Media und Internet. Forschungslage und medienpädagogische Implikationen

Herausgegeben von Kai Hugger, Kai Kaspar und Lars Gräßer

\section{Kreative Bearbeitung politischer Information auf TikTok}

\section{Eine multimethodische Untersuchung am Beispiel des Hashtags \#ww3}

Judith Ackermann und Leyla Dewitz

\begin{abstract}
Zusammenfassung
Der vorliegende Artikel befasst sich mit politischer Information auf der Mikro-Video-Plattform TikTok ausgehend von dem Hashtag \#ww3, welches im Januar 2020 im Zusammenhang mit einem vermeintlichen Ausbruch eines 3. Weltkriegs grosse Popularität erhielt, nachdem US-Präsident Donald Trump einem von den USA geführten Drohnenangriff zugestimmt hatte, bei dem Irans General Qassem Soleimani getötet wurde. Auf Basis von 1373 entsprechend verschlagworteten Videos inklusive Captions wird multimethodisch analysiert, inwiefern Nutzende die unterschiedlichen kommunikativen Ebenen, die das Netzwerk bereit hält, verwenden, um - ausgehend von einem konkreten Thema - persönliche Bezüge, Gedanken und Einstellungen zu diesem zu verhandeln. Die quantitative Auswertung zeigt, dass der Wunsch nach Viralität und Sichtbarkeit zwar einen hohen Stellenwert für Nutzende auf TikTok hat, Videos und Captions aber auch intensiv genutzt werden, um sich mit politischen Themen und Fragestellungen auseinanderzusetzen. Auf welche Weise dies geschieht, wird im Rahmen des qualitativen, inhaltsanalytischen Teils der Studie betrachtet. Als gestalterische Mittel zur kreativen Bearbeitung politischer Information lassen sich dabei u.a. die Transformation bzw. der Plot-Twist in der Narration der Videos sehen, die semantische Klärung durch Text-Insertierungen, der Wunsch, eine primäre Lesart für das eigene Video vorzuschlagen und die ironische Überhöhung zur Distanzierung von eigenem Content oder emotionalen Triggern.
\end{abstract}

Creative appropriation of political information on TikTok. A multi-method investigation of the hashtag \#ww3

\begin{abstract}
This paper deals with political information happening on the micro video platform TikTok based on the hashtag \#ww3, that got popular in January 2020 with regard to the question, if a third world war could break out, since US President Donald Trump agreed to a US-led drone attack that killed Iran's General Qassem Soleimani. Based on 1373 videos and captions tagged with \#ww3 the study analyses in how far users employ the
\end{abstract}


different communication possibilities of the network to negotiate personal connections, thoughts and opinions associated with \#ww3. A quantitative research shows that the desire of being visible and getting viral on the platform must be seen as the primary motivation in producing content on TikTok. Still videos and captions are also intensely used to deal with political topics and questions. A qualitative content analysis reveals that appropriating political information by producing TikToks among others focuses on including transformations and plot twists into a narration, applying text inserts into the videos for semantic clarification, promoting a dominant way of understanding a video as well as using ironical exaggerations to increase the distance between a user and their content.

\section{Einleitung}

Politische Information erreicht ihre Adressatinnen und Adressaten nahezu ausschliesslich medial vermittelt. Soziale Medien lassen sich in diesem Kontext als "Quelle bzw. Mittel politischer Information wie als Gelegenheitsstruktur für politische Kommunikation bzw. Interaktion und Partizipation» (Wagner und Gebel 2014, 2) gleichermassen einordnen. Im Kontext des aktuellen Medienensembles bieten digitale Plattformen aufgrund der Möglichkeit zur Quasi-Live-Publikation von Information und Nachrichten besondere Chancen und Dynamiken. Gerade in sozialen OnlineNetzwerken versammeln sich die unterschiedlichsten Akteurinnen und Akteure aus dem Feld der politischen Information, die entsprechende Inhalte sowohl platzieren als auch rezipieren, überformen und reartikulieren. Dabei lassen sich mit Wagner und Gebel $(2014,2)$ «Rezeption und Produktion von Information mit gesellschaftlicher Relevanz [...] [gleichermassen] als Teil der politischen Kommunikation im weiteren Sinn» dem Verständnis von Schulz $(2011,16)$ folgend einordnen. In diesem Zusammenhang findet immer auch eine, wie bereits 2001 von Meyer $(2001,10)$ beschriebene «Unterwerfung der Regeln des Politischen unter die Regeln des Mediensystems» statt. Anders als im klassischen Nachrichten-Journalismus und Öffentlichkeitsarbeits-System bergen Soziale Online-Netzwerke das Potenzial, die Hierarchien zwischen den einzelnen Akteurinnen und Akteuren aufzulösen. Macht und Sichtbarkeit werden in Followerinnen- und Followerzahlen und Reichweite übersetzt. Algorithmen entscheiden auf Basis von Bekanntheit und Nutzendenvorlieben darüber, wer zu welchem Zeitpunkt welche Information zu sehen bekommt. In Bezug auf die Bedeutungsproduktion können unterschiedliche Lesarten politischer Informationen so potenziell gleichermassen in den Fokus rücken.

Im Sinne der Medienaneignung lassen sich auf digitalen Plattformen die kommunikativen Aneignungsprozesse der Einzelnen in mediatisierter Form in das Medienensemble zurückspielen. Die Sichtbarkeit und Verbreitung der verschiedenen Kommunikate kann durch exzessive Teilungspraktiken und Unterstützungsbekundungen 
(etwa durch Likes, Kommentare und Follows) wiederum zu dominanten Lesarten innerhalb der Aneignungsprozesse führen. Entsprechend des Aneignungskonzepts werden «Medienbedeutungen [...] nicht einfach übernommen, sondern im Kontext von Alltagserfahrungen, die an die Medientexte herangetragen werden, verhandelt und produktiv angeeignet.» (Röser und Peil 2010, 221).

Ausgehend von dem Hashtag \#ww3 beschäftigt sich der vorliegende Artikel mit politischer Information auf der Mikro-Video-Plattform TikTok. Dort «trendete» das Hashtag im Januar $2020 \mathrm{im}$ Zusammenhang mit der Debatte um einen vermeintlichen Ausbruch eines 3. Weltkrieg, nachdem US-Präsident Donald Trump einem von den USA geführten Drohnenangriff zugestimmt hatte, bei dem der iranische General Qassem Soleimani getötet wurde. Auf Basis von 1373 entsprechend verschlagworteten Videos inklusive Captions, wird multimethodisch analysiert, inwiefern Nutzende die unterschiedlichen kommunikativen Ebenen, die das Netzwerk bereit hält, verwenden, um, ausgehend von einem konkreten Thema, persönliche Bezüge, Gedanken und Einstellungen zu diesem zu verhandeln. "Politische Information» wird im Kontext dieses Artikels als erweiterter Begriff verstanden, der sich auf den Umgang, die Aneignung und Weitergabe von Kommunikaten mit politischem Informationsgehalt bezieht. Politische Information wird dabei nicht implizit mit einem objektiven Wert und Wahrheitsgehalt gleichgesetzt, sondern vielmehr als eine Botschaft verstanden, die von den Sendenden zu den Empfangenden weitergegeben wird, wobei das Medium und die Art der vermittelten Information nicht ausschliesslich durch Objektivität gekennzeichnet und auf mediale Nachrichten beschränkt sein müssen. Die mit dem Hashtag \#ww3 versehenen Inhalte zeigen eine massive Diffusion von Memes, die Emotionen transportieren. Nach Dawkins (1976), repräsentieren Memes kulturelle Entitäten, die erstellt wurden, um (kulturelle) Ideen zu replizieren, zu transformieren, zu reproduzieren und zu teilen, sowie ihren Produzierenden zu ermöglichen, individuelle Bedeutungen und/oder Akzente hinzuzufügen. Angewandt auf unseren Kontext, erlauben Memes den Nutzenden, ihre persönlichen Einschätzungen von politischen Situationen und ihren möglichen Folgen - im Zusammenhang mit \#ww3 etwa einem möglichen Kriegsausbruch - abzuhandeln und ihre Emotionen basierend auf der kreativen Reproduzierbarkeit von Bild- und Videoinhalten auszudrücken. Die Untersuchung konzentriert sich auf die App TikTok, da diese durch die strenge Limitierung der für die erstellten Inhalte zur Verfügung stehenden Zeit und den Fokus auf fiktionale Inhalte in Bezug auf die Verhandlung politischer Themen eine hohe Prägnanz in der Umsetzung erfordert und grosse Kreativität in der Aneignung zulässt. 


\section{Aktueller Forschungsstand zu TikTok}

Das Prinzip der Social-Media-Plattform TikTok ${ }^{1}$ (ehemals musical.ly; Douyin in China) basiert darauf, eigene Mikro-Videos von, in der Regel, bis zu 15 Sekunden zu gestalten und zu teilen, die hauptsächlich auf Lipsync- und Tanz-Elementen zu bestehenden Sound-Samples basieren, aber auch vollständig selbst erstellt werden können. Von Nutzenden produzierte Tonspuren können dann von weiteren Userinnen und Usern wiederum zur Hinterlegung eigener Videos verwendet werden. Die 2017 gegründete Plattform ist aktuell die am schnellsten wachsende Social-Media-Anwendung der Welt und führte die Rangliste der «Most Downloaded Apps» in den USA im Jahr 2018 an. Bisher ist TikTok in 150 Ländern verfügbar und soll mehr als 500 Millionen aktive Nutzende haben (Bo 2018; Yeh 2019; Winkler 2019). Im Vergleich zu anderen SocialMedia-Plattformen zeichnet sich TikTok durch seinen Fokus auf Bewegtbild aus, welches mit einfach zu verwendenden Bearbeitungsmethoden, wie Schnittverfahren, Insertierung von Text und Hintegrundbildern und dem Hinzufügen spezieller Effekte, eine unkomplizierte Form der Erstellung von Inhalten ermöglicht. Im Vergleich zu länger bestehenden Plattformen wie Facebook, Twitter und Instagram ist der Anteil institutioneller Accounts auf TikTok derzeit noch deutlich geringer. Im Bereich Nachrichten und politische Bildung in Deutschland präsentiert sich das ZEIT Magazin seit Oktober 2019 auf TikTok (200 Followerinnen und Follower) ${ }^{2}$, die Tagesschau bespielt seit November 2019 ebenfalls einen eigenen Kanal (ca. 490.000 Followerinnen und Follower) ${ }^{3}$. Das Gesundheitsministerium ist seit Ende März 2020, befördert durch Kommunikationsanliegen im Zusammenhang mit der Corona-Pandemie, ebenfalls mit einem Kanal (ca. 55.000 Followerinnen und Follower) ${ }^{4}$ auf TikTok vertreten. Accounts deutscher Parteien, sowie von führenden Politikerinnen und Politikern sind mit Stand vom Mai 2020 kaum präsent. Politische Informationen werden daher zu grossen Teilen von Privatnutzenden selbst auf der Plattform platziert, verhandelt und mit Bedeutung versehen. Gleichzeitig gestatten die vorhandenen institutionellen Accounts, durch das Hochladen von Videos mit eigenen Sounds, der Community die Verwendung der jeweiligen Tonspuren für ihre eigenen Inhalte und damit die Verbreitung und Präsentation ihrer Deutungen und Ideen zu spezifischen Inhalten, welche je nach Reichweite stark skalieren können.

Die Diffusion von Internet-Memes zeigt ein hochdynamisches Phänomen der Kommunikation im Zusammenhang mit einem bestimmten Thema oder einer Diskussion. Shifman (2014) wies bereits auf das polysemische Potenzial von Memes hin, das auf die Offenheit des visuellen Materials und das Fehlen eines klaren Narrativs zurückzuführen ist und verschiedene Interpretationen zulässt. Das Auftauchen von

\footnotetext{
https://www.tiktok.com/de/ (08.05.2020).

https://www.tiktok.com/@zeitmagazin (08.05.2020).

https://www.tiktok.com/@tagesschau (08.05.2020).

https://www.tiktok.com/@bmg_bund (08.05.2020).
} 
ton-basierten Memen auf TikTok, die im Allgemeinen eine Lücke zwischen der dargestellten Bedeutung eines bestimmten Tons (Elements) und seiner audiovisuellen (Re-)Interpretation mittels selbst produzierter Videos schaffen, birgt das Potenzial für eine noch grössere Pluralität von Ausdrucksmöglichkeiten. In dieser Perspektive kann die Logik der Plattform TikTok als wesentliche Beeinflusserin des kreativen Outputs betrachtet werden, der sich, in den von den Nutzenden generierten Inhalten, widerspiegelt.

Ein besonders hervorstechendes Merkmal von TikTok ist die Schnelligkeit, in der Videos trenden. Dies wird oft als virales Phänomen bezeichnet, in dem bestimmte Videos durch die hohe Frequenz der Share- und Like-Aktivitäten populärer werden als andere. Die Viralität eines Videos wird als ein Mass für den Erfolg von Nutzenden auf Plattformen gesehen und ist oft beeinflusst durch die Anzahl der Followerinnen und Follower und damit der Reichweite. Trendende Videos auf TikTok ziehen Tausende von Kommentaren und Millionen von Nutzenden an, die ihre Vorlieben und Abneigungen verbalisieren und damit zum Erfolg oder Misserfolg eines Videos beitragen. Welche Videos von der Plattform selbst gefördert werden und warum bzw. wie der TikTok-Algorithmus funktioniert, wird nicht offengelegt (Omar und Dequan 2020).

Trotz der immensen Popularität TikToks gibt es momentan nur wenige Studien, die sich auf Phänomene der Video-Sharing-App beziehen (Literat und KliglerVilenchik 2019). Dabei zeigt die Plattform aufgrund der rasant steigenden Anzahl von Nutzenden und anhaltenden kritischen Stimmen zu Jugendschutz, Datenschutzregularien und Zensurpraktiken einen hohen Bedarf an Auseinandersetzung (u.a. Beutell 2020; Feld 2019; Smolentceva 2019; Köver 2020; Köver und Laufer 2020; Knuth 2019; Zolfagharifard 2019). Ein Grund für den wachsenden Erfolg der Plattform wird vor allem in der Nutzung von Videos, Musik und aktiven Elementen (Tanz) gesehen, die sich im Vergleich zu anderen videobasierten Social Media Plattformen wie YouTube einfacher erstellen und mit speziellen Effekten versehen verbreiten lassen. Damit hebt sich TikTok von allen anderen Social Media Plattformen ab und setzt auf die kreativen Potenziale der meist sehr jungen Nutzenden (Herrman 2019). Das Herausarbeiten plattformabhängiger Merkmale und Phänomene ist für medien- und musikpädagogische Fragestellungen daher von hohem Interesse (Höfer 2019; Müller 2019).

In einer Studie befragten Omar und Dequen (2020) $N=385$ Userinnen und User zu ihrem Nutzungsverhalten auf TikTok. Unter Anderem zeigte sich, dass Social Media Phänomene, wie das Konsumieren (browsen, beobachten, lesen oder ansehen), Beteiligen (liken, hinzufügen, teilen, posten und kommentieren) und Produzieren (erstellen und veröffentlichen persönlicher Inhalte) von Inhalten auf TikTok noch viel stärker vertreten sind als in vergleichbaren sozialen Medien. Dies wird damit begründet, dass die Erstellung von Inhalten bei TikTok enger mit der Aneignung und Reproduktion von Inhalten anderer Nutzender verbunden ist. So ist etwa das intensive Konsumieren von Trends bzw. Videos notwendig, um einen Trend nachzuahmen 
und/oder Viralität zu erreichen. Ein zentrales Ergebnis dieser Studie war zudem, dass Probandinnen und Probanden motivierter waren, sich durch das Liken, Teilen und Kommentieren von Inhalten anderer Nutzender aktiv zu beteiligen und zudem eigene Videos zu produzieren, als lediglich passiv Videos Anderer zu konsumieren. Dieses Ergebnis steht nach Omar und Dequan (2020) in Kontrast zu anderen Social Media Plattformen wie Facebook und Instagram, bei denen sich vorwiegend passive Verhaltensweisen im Zusammenhang mit dem Nutzungsverhalten zeigen und eher selten aktiv Inhalt erstellt wird.

Das Motiv für die Nutzung TikToks liegt nach Wang (2020) im Erleben eines Gefühls von Immersion, sozialer Präsenz und Unterhaltung. Soziale Präsenz vermittelt dabei indirekt einen Zusammenhang zwischen Selbstdarstellung und der Absicht der Zuschauenden beim Anschauen der Videos und damit deren Verbreitung. Zuo und Wang (2019) identifizieren TikTok als popkulturelles Phänomen, in dem Nutzende stilistisch mit Humor, Übertreibung und Ironie spielen. Die Selbstdarstellung ist dabei ein hervortretendes Merkmal. Das unterstreicht auch die Studie von Khattab (2020), welche inhaltsanalytisch 2750 TikTok-Videos untersucht, um zu veranschaulichen, wie neue digitale Medien stereotype Körperbilder von Schönheit und Geschlecht in Bezug auf Performativität und Selbstdarstellung repräsentieren. Die Ergebnisse zeigen, dass sich die Darstellung des Körpers vor allem auf geschlechtsspezifische Bilder von heteronormativer Männlichkeit und Weiblichkeit sowie auf die Wahrnehmung von Schönheit als Indikator für sexuelle Anziehungskraft bezieht.

Im Hinblick auf Kommunikation politischer Informationen auf TikTok finden sich derzeit nur wenige Forschungsbeiträge. Die Frage, ob die Vorgänger-Plattform musical.ly im Hinblick auf politische Meinungsäusserung von Bedeutung ist, adressieren Literat und Kligler-Vilenchik (2019) in ihrer Studie, die $N=1651$ Datensätze bestehend aus Videos und Hashtags von Jugendlichen im Kontext der politischen Wahlen in den USA 2016 analysiert. Die entsprechenden Ergebnisse verdeutlichen, dass die Plattform neue Formen politischen Ausdrucks unter Jugendlichen aufzeigt und als ein Ort der politischen Meinungsäusserung zu identifizieren ist. Zudem wurden unzählige kreative Formen der Vertretung politischer Positionen erkannt, die durch die Vergabe von Hashtags kodiert wurden. Diese wurden verwendet, um sich einer (kollektiven) politischen Meinungsäusserung anzuschliessen oder sich von dieser abzugrenzen. Durch die Vergabe entsprechender Hashtags (etwa \#makeamericagreatagain oder \#notmypresident) wurden Nutzende Teil eines grösseren Diskurses.

Auch die Studie von Serrano et al. (2020) untersucht die politische Kommunikation auf der Plattform und stellt fest, dass das Medium immer häufiger dazu genutzt wird, politische Meinungen zu teilen. Im Rahmen der Studie wurden N=5946 Videos von Mitgliedern der US-amerikanischen republikanischen und demokratischen Parteien untersucht, um herauszufinden wie die Nutzenden miteinander kommunizieren. So konnte u.a. festgestellt werden, dass Parteimitglieder der Demokraten eher 
mit politischen Gegnerinnen und Gegnern interagierten, z.B. im Rahmen von Duetten, als Republikanerinnen und Republikaner. Auf Basis einer Hashtag-Analyse wurde zudem ermittelt, dass die Nutzung von Hashtags mit politischen Inhalten auf TikTok stärker verbreitet ist als auf der Plattform Instagram und zudem interaktiver ist, da TikTok-Nutzende differenziertere Möglichkeiten der Interaktion und Kommunikation haben, um ihre Botschaften zu verbreiten.

\section{Methodisches Vorgehen}

Die vorliegende Studie fokussiert die Kommunikation politischer Information auf der Video-Sharing-Plattform TikTok am Beispiel der Verbreitung des Hashtags \#ww3. Dieser wurde Anfang Januar 2020 verstärkt verwendet, nachdem bekannt worden war, dass der US-Präsident Donald Trump die Ermordung des iranischen Generals Qassem Soleimani angeordnet hatte. Das Hashtag entwickelte sich als Reaktion auf den aufkeimenden politischen Konflikt und trendete mehrere Tage auf Social-MediaPlattformen (Müller 2020). Vor allem auf TikTok zeigte die Verwendung von \#ww3 vielfältige Varianten kreativer Aneignung und Reproduktion von Bild- und Videoinhalten als eine Art Bewältigungsstrategie (Steadman und Brooks 2020; Romano 2020; Rezo 2020). Mediale Aufmerksamkeit bekam das Hashtag in Deutschland vor allem durch ein TikTok-Video, das am 5. Januar veröffentlicht wurde. Es zeigt die 18-jährige deutschsprachige Influencerin Laura Sophie (2,2 Mio. TikTok-Followerinnen und -Follower), wie sie die politische Situation zwischen der Islamischen Republik Iran und den Vereinigten Staaten von Amerika aus ihrer Sicht erläutert. Das Video, in dem sie unter anderem Erleichterung darüber äussert, dass Deutschland diesmal nicht an einem Weltkrieg schuld sei, verbreitete sich viral. Nach einer grossen Welle der Kritik, entfernte Laura Sophie das Video und entschuldigte sich öffentlich (Dettwiler und Stauffacher 2020; Petter 2020; Weber 2020; Weichert 2020). Unter dem Hashtag wurden jedoch zahlreiche Videos anderer Nutzenden gepostet. Das Beispiel kann als prototypisch für die Dynamik angesehen werden, mit welcher die Aushandlung von (politischen) Informationen zwischen unterschiedlichsten Akteurinnen und Akteuren auf TikTok stattfinden kann und sich bar jeglicher Ansprüche an eventuellen Wahrheitsgehalt oder Prüfung verstärkt ausgehend von Fragen ihrer Ästhetik und Emotionalität weiter reproduziert und transformiert. Um in diesem Feld kompetent agieren zu können ist es für die Medienpädagogik entsprechend zentral, zu verstehen, nach welchen Verfahren die gemeinschaftliche kreative Aushandlung und Aneignung politischer Informationen in diesem Kontext erfolgt.

Am Beispiel der Verbreitung von \#ww3 greift diese Arbeit die Interaktion mit politischer Information auf TikTok auf Basis folgender Fragestellungen auf:

- Welche Inhalte werden unter dem Hashtag \#ww3 geteilt?

- Welche dieser Inhalte lassen sich als kreative Bearbeitung politischer Information identifizieren? 
- Welche Arten von Hashtags tauchen in Kombination mit \#ww3 am häufigsten auf?

- Wie unterstützen Videounterschriften (Captions) die kreative Bearbeitung der politischen Information?

- Wie wird die politische Information auf audiovisueller Ebene vermittelt?

Zur Beantwortung der Fragen wurden 1.500 Videos, die unter dem Hashtag \#ww3 gepostet wurden, von öffentlich zugänglichen Profilen der Plattform TikTok, ausgelesen ${ }^{5}$. Über den Web-Zugang von TikTok konnte mithilfe eines Skriptes basierend auf Node.js am Tag des 30.01.2020 von ca. 20:06 Uhr bis 20:41 Uhr historisch auf Posts im Zeitraum vom 3. Januar bis 29. Januar 2020 zugegriffen werden. Die ausgelesenen Daten bestehen aus Video- und Caption-Inhalten, Musik-IDs sowie Nutzenden-Informationen und wurden im mp4-Format gespeichert bzw. in eine csv-Datei in Microsoft Excel transferiert und auf einer verschlüsselten Festplatte gesichert. Nach Datenbereinigung enthält die zu untersuchende Stichprobe ${ }^{6} N=1373$ Datensätze und beinhaltet Videos und dazugehörige Captions (Videounterschriften mit Hashtags, Emojis und Verlinkungen). Die Datenbereinigung erfolgte nach Parametern der Verständlichkeit der Dateninhalte: alle Datensätze der csv-Datei, die nicht englisch- oder deutschsprachig waren, wurden demnach aus dem Datenset entnommen (insgesamt 127 extrahierte Datensätze). Dies wurde aus Gründen der späteren Interpretierbarkeit der Dateninhalte als notwendig angesehen.

\subsection{Quantitative Analyse der Hashtags}

Als Basis für die quantitative Analyse, wurde in einem ersten Schritt induktiv aus den verwendeten Hashtags des empirischen Materials, ein Kategoriensystem entwickelt. Dies geschah um feststellen zu können, welche Funktionen dieses Element im Rahmen des kommunikativen Handelns auf TikTok erfüllt. Für die Analyse wurden alle Hashtags des Samples berücksichtigt, die mindestens zweimal verwendet wurden (419 distinkte Hashtags mit 8065 Vorkommen). Als erste Kategorie wurde (1) concrete topic identifiziert, welche neben dem Hashtag \#ww3 alle diesen Ausdruck beinhaltenden und ergänzenden Hashtags umfasst, sowie solche, die mit Variationen der Schreibweise und leichten Abwandlungen arbeiten. Eine weitere zentrale Kategorie stellt (2) virality \& visibility dar. In dieser werden alle Hashtags zusammengenommen, die darauf abzielen, die Wahrnehmung des eigenen Videos zu erhöhen. Dies sind vor allem Hashtags, die \#foryou beinhalten bzw. in unterschiedlichen Schreibvarianten realisieren, sowie alle, welche die Begriffe \#viral, \#feature und \#trend beinhalten und/oder auf die Plattform selbst referenzieren (z.B. \#tiktok). Auffällig in dieser Kategorie ist, dass die Hashtags teilweise als direkte Aufforderungen formuliert

5 TikTok Scraper auf GitHub: https://github.com/drawrowfly/tiktok-scraper (16.11.2020).

6 Erklärung zur Stichprobenziehung und Sampling sind dem Kapitel Limitationen der Studie zu entnehmen. 
sind, das eigene Video erfolgreich werden zu lassen (\#featurethis, \#makemefamous, \#dontletthisflop). Als weiteren Schwerpunkt liessen sich (3) popcultural references ausmachen, welche insbesondere Verweise auf Games, Filme und Animes, aber auch auf populäre Influencerinnen und Influencer sowie berühmte Persönlichkeiten beinhalten. Darüber hinaus wurden (4) aesthetics \& ways of production häufig verschlagwortet, etwa durch Verweise auf verwendete Filter (\#greenscreen, \#megamouth) oder spezifische Challenges (\#handgestures, \#jumanjichallenge), und (5) reception \& meaning, welche Hinweise zu möglichen Lesarten der Videos geben (etwa \#funny, \#sad oder \#fail). Die grössten Variationen finden sich in der Kategorie (6) topic elaboration \& connections, die von den Nutzenden verwendet wird, um das Hauptthema weiter auszubauen oder damit - mehr oder weniger - lose verbundene weitere Themen zu besprechen. Zusätzlich finden sich Hashtags aus den Bereichen (7) sports, (8) school und (9) animals sowie (10) seasonal, (11) commercial und (12) other. Ein Überblick über die anteiligen Vorkommen der Kategorien in den einzelnen Hashtags kann Abb. 1 entnommen werden.

Prozentuale Verteilung der Kategorien

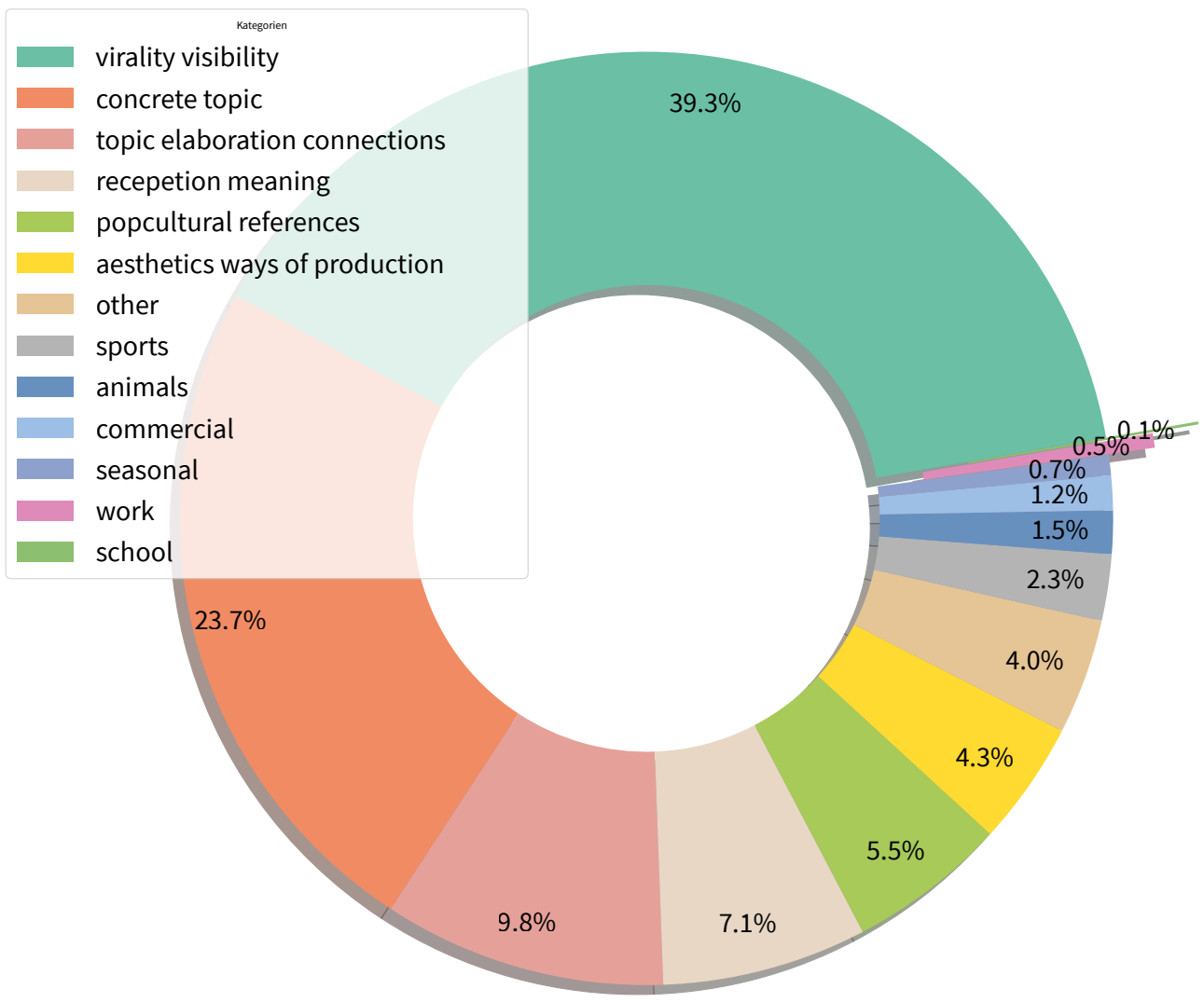

Abb. 1.: Prozentuale Verteilung der Kategorien von Hashtags $(\mathrm{N}=8065)$. 
Rückübertragen auf die Gesamtheit zeigt sich, dass die einzelnen Kategorien unterschiedlich häufig miteinander in Kombination auftreten (s. Abb. 2). Mit 86,6 \% der Fälle ist die Kombination der Ausgangskategorie concrete topic mit der Kategorie virality \& visibility die mit Abstand am häufigsten auftretende. Danach folgen ungefähr gleich stark vertreten Kombinationen mit reception \& meaning $(32,0 \%)$ sowie mit topic elaboration \& connections (31,6\%). In 20,8\% der Fälle tauchen Kombinationen zu popcultural references auf, in 20,5\% zu aesthetics \& ways of production und in 19,4\% zu other. Kombinationen mit der Kategorie sports machen 10,0\% der Fälle aus. Alle übrigen Kombinationen befinden sich im einstelligen Prozentbereich (animals, 8,0\%; commercial, 7,3\%; seasonal, 4,0\%; work, 3,0\% und school unter 1,0\%). Die Verteilung verweist deutlich darauf, dass «Viralität und Sichtbarkeit» als besonders wertvolles Gut in der TikTok-Kommunikation betrachtet wird.

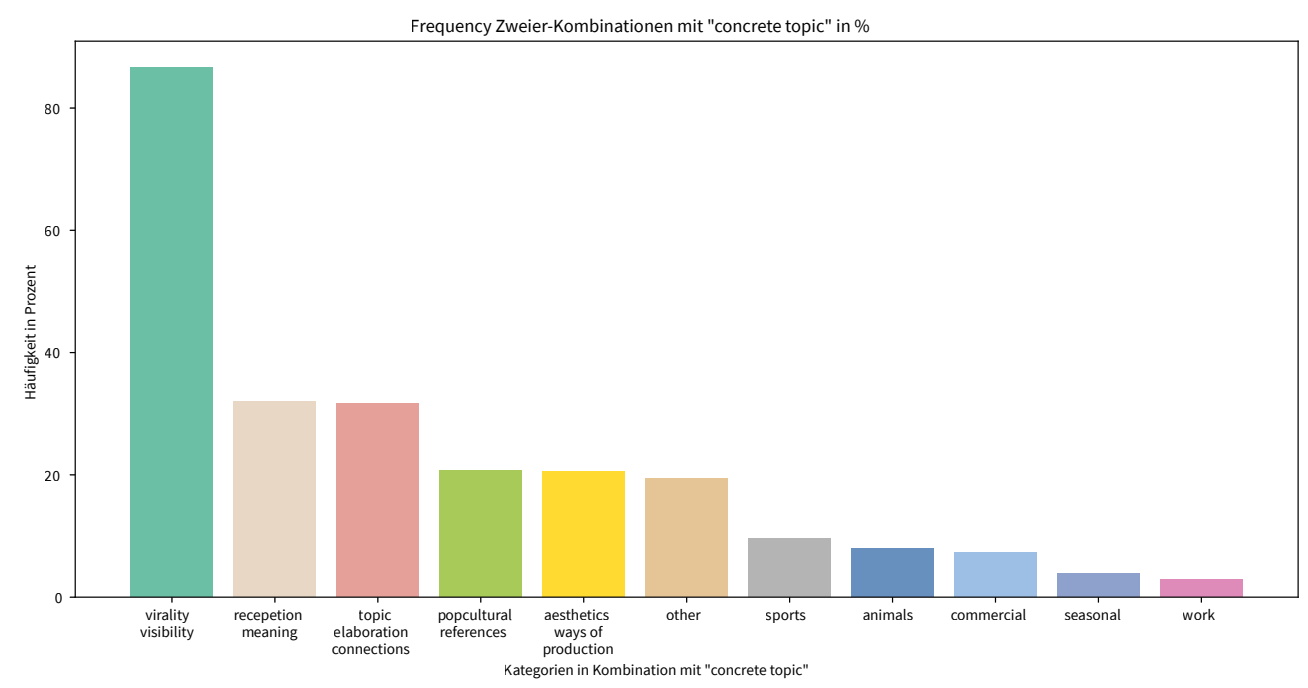

Abb. 2.: Prozentuale Verteilung der Zweier-Kombinationen der Kategorien von Hashtags $(\mathrm{N}=8065)$.

Dieser Eindruck verstärkt sich nochmals bei Betrachtung von Dreierkombinationen (siehe Abb. 3). Hier beinhalten die Kombinationen mit dem höchsten Vorkommen alle die Verbindung von Hauptkategorie und virality \& visibility und ergänzen diese insbesondere um die Bereiche reception \& meaning $(29,4 \%)$ und topic elaboration \& connections (27\%). An dritter und vierter Stelle folgen Kombinationen mit aesthetics \& ways of production $(18,1 \%)$ oder popcultural references $(17,6 \%)$. Die Betrachtung der Dreierkombinationen zeigt, dass nach der Verschriftlichung des Wunsches nach Viralität, die Mitgabe einer Lesart und die Verhandlung von durch das Hauptthema initialisierten Themen besonders zentral zu sein scheinen. 


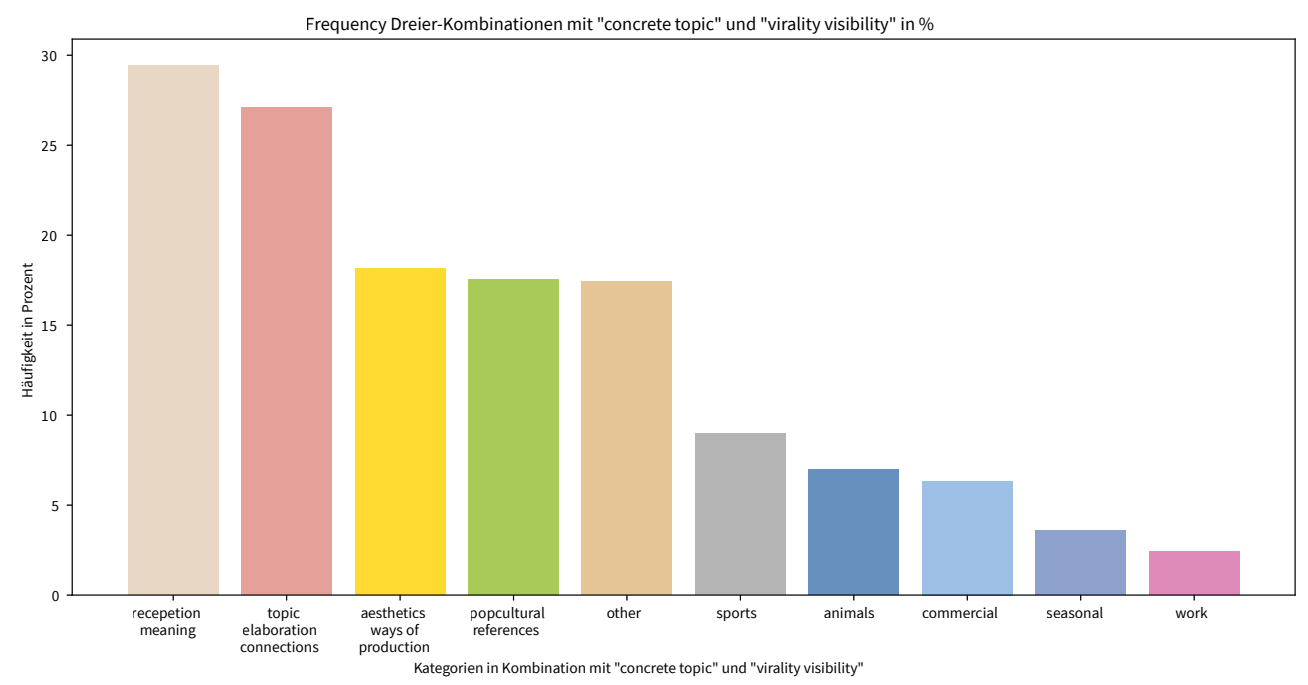

Abb. 3.: Prozentuale Verteilung der Dreier-Kombinationen der Kategorien von Hashtags $(\mathrm{N}=8065)$.

Die mitverhandelten Themen sind dabei vielfältig. In der Folge sollen exemplarisch zwei von ihnen näher betrachtet werden: Zum einen (A) vermutete Auswirkungen eines möglichen Kriegsausbruchs auf die Rollenverteilung und das Geschlechterverständnis in der Gesellschaft und zum anderen (B) die Auseinandersetzung mit der die eigene Person betreffenden Sorge, im Falle eines möglichen Krieges, selbst eingezogen zu werden. Beide Themen werden auf zwei unterschiedlichen Ebenen einer qualitativen Betrachtung unterzogen: Im ersten Schritt werden die Captions der Videos auf zugehörige Beschreibungen und/oder Hashtags hin analysiert, für den zweiten Schritt werden mit den beiden Thematiken besonders verbundene Sounds identifiziert und die dazu erstellten Videos einer qualitativen Betrachtung unterzogen. In einem dritten Schritt werden die Ergebnisse aufeinander abgebildet.

\subsection{Qualitative Analyse der Captions}

Alle Informationen und Metainformationen (Specs - specifications), d.h. nicht-videobasierten Elemente eines Posts, wie die Caption (Videounterschrift), Erwähnungen (Verlinkungen von anderen Nutzenden) und vergebene Hashtags sind bei TikTok begrenzt durch ein Limit von max. 150 Zeichen. Die Captions enthalten meist Informationen zur Verdeutlichung des geposteten Contents. Zudem helfen sie beim Erzeugen von Viralität durch das Setzen von Hashtags z.B. zu Trends und Challenges. Die Captions enthalten darüber hinaus meist text- oder hashtag-basierte Ausdrücke von Emotionen (u.a. Wut, Trauer, Freude, Entsetzen). Diese Emotionen werden oft durch die Vergabe von Emojis entweder linear zum Inhalt des Textes oder gegensätzlich dazu akzentuiert (z.B. beim Einsatz von Stilmitteln wie Ironie und Humor). 
Im Rahmen der Caption-Analyse konnten 31 Captions identifiziert werden, die in Zusammenhang mit geschlechtsspezifischen Bildern von Männlichkeit und Weiblichkeit stehen und sich auf wahrgenommene Vorstellungen zu Geschlechterrollen und der Verhandlung von Stereotypen konzentrieren. Es konnten zudem insgesamt 175 Captions identifiziert werden, in denen die Rekrutierung oder die Einberufung bzw. das Eingezogen werden (engl.: draft) zum Militärdienst thematisiert wird. Innerhalb dieser 175 Captions wurden 72 Hashtags identifiziert, die den Wortstamm \#draft beinhalten.

Im Rahmen der qualitativen Analyse konnte, durch ein methodologisch inhaltsanalytisches Vorgehen nach dem Ansatz von Krippendorf (2019), induktiv ein Kategoriensystem entwickelt werden. Innerhalb der Hauptkategorie Gender liessen sich folgende Unterkategorien identifizieren: (A1) Geschlechterrollen und Gleichberechtigung (binär/non-binär) und (A2) Frauen und Militärdienst, welche gleichermassen auch als Teilbereich (B1) der Hauptkategorie Eingezogen werden zugeordnet werden kann. Als weitere (eigenständige) Subkategorien im Bereich «Eingezogen werden» konnten (B2) Vorbereitung auf die eigene Rekrutierung, (B3) das Moment der Rekrutierung und (B4) die Verhinderung der eigenen Rekrutierung, identifiziert werden. In der Folge werden die einzelnen Subkategorien der zwei Bereiche anhand von Beispielen näher erläutert.

\section{(A1) Geschlechterrollen und Gleichberechtigung (binär / non-binär)}

Ein Phänomen, das sich aus den Daten heraus zeigte, ist die Verhandlung von binären Geschlechterrollenklischees. Dabei reichen die Captions von Ironie bis Ernsthaftigkeit und thematisieren die Rolle und Aufgaben der Frau bzw. Hausfrau und Mutter während der Mann im Krieg kämpft:

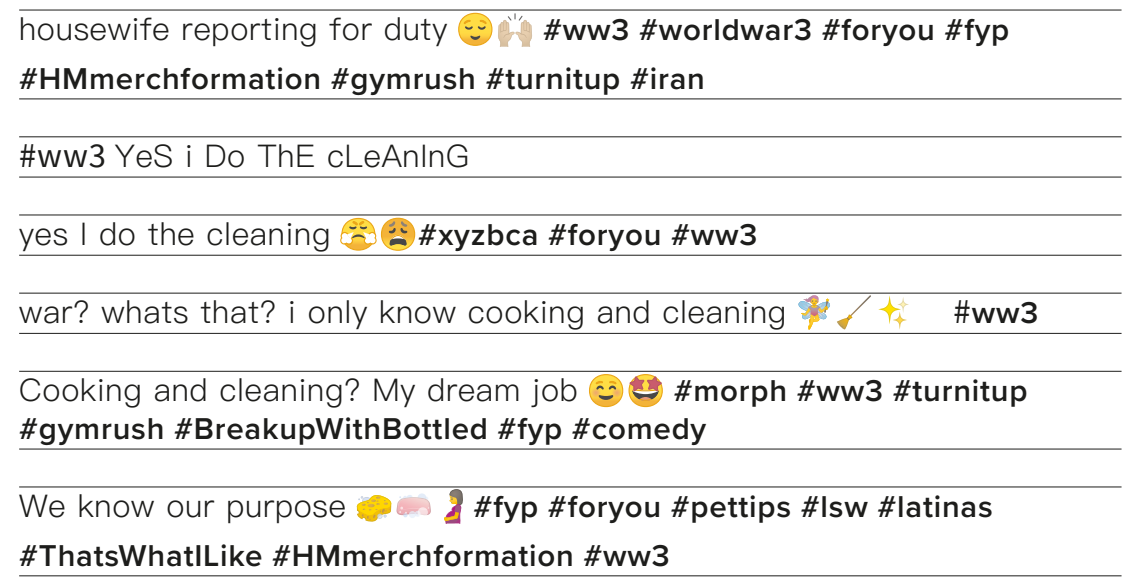


Mothers will do anything for their babies \#fyp \#foryou \#foryoupage \#viral

\#ww3 \#moms \#momsbelike

mother's duty is never done @littlesehgals \#mom \#foryou \#ww3

\#foryoupage \#ThatsWhatlLike \#fyp \#iykyk \#relatable \#laugh \#funny \#4u

\#fy \#ww3 \#ww3 \#ww3

And that's on MOTHERHOOD $\approx$ \#momlife \#momsoftiktok \#fyp \#foryou

\#ww3 \#itwentlike \#ThatsWhatlLike \#foryoupage

Innerhalb der Kategorie zeigt sich zudem die direkte Thematisierung von Gleichberechtigung und die Verhandlung von Aufgaben, die möglicherweise mit einer ironischen Auseinandersetzung der Gleichbehandlung der Geschlechter verbunden sind:

equality??? idk her the womans place is in the kitchen!!!!! \#fyp \#foryou

\#foryoupage \#women \#ww3

no more equality \#fyp \#foryou \#wwiii \#worldwar3 \#ww3 \#gymrush \#turnitup \#ThatsWhatlLike \#womenpower \#BreakupWithBottled \#Imao \#dog \#dothedishes

They forgot that equal rights meant equal responsibilities. \#foryou \#fyp \#4up \#viral \#comedy \#wwiii \#ww3 \#femenist \#woman

Equal rights?who is she? $\odot$ \#ww3 \#fyp

women's rights all the way but... i'll be here minding my business (: \#ww3 \#fyp \#foryou \#lsw \#pettips

equality; am i wrong? hahaha \#dlaciebie \#foryoupage \#wwiii \#wwiimemes \#ww3 \#global

Innerhalb der Kategorie finden sich zudem Beispiele der Ablehnung von NonBinarität und der Verteidigung klassischer Geschlechterrollen:

just leave the gays at home. we can cook and clean your houses. \#ww3

\#fyp

There are two genders. \#sorrynotsorry \#gender \#dishwasher \#twogenders \#trump \#trump2020 \#pilotpete \#perfectmeal \#petdrip

\#StraightToHell \#ww3 
$\left(A 2^{\wedge} \mathrm{B} 1\right)$ Frauen und Militärdienst

Ein Phänomen, das sich vermehrt innerhalb der Captions zeigte, ist die Erwähnung des Militärdienstes im Zusammenhang mit \#ww3. Dabei behandeln die Captions die Auseinandersetzung mit dem Eingezogen bzw. Rekrutiert werden für einen anstehenden Kriegseinsatz. In der Kategorie «Gender» drückt sich das vor allem in der Thematisierung der eigenen Rolle als Frau aus:

\begin{tabular}{l}
\hline Please don't let women be drafted; I don't wanna fight. \#ww3 \#wwiii \\
\#usa \#fyp \#foryou \#foru \#foryoupage \#turnitup \#ThatsWhatlLike \#foruyou \\
\hline doing a woman's job so I don't have to get drafted for ww3 \\
\#fyp \#viral \#ThatsWhatlLike \#ww3 \#womansjob \#turnitup \\
\hline you can't make pregnant women fight \#xyzbca \#ww3 \#tiktokww3 \\
\#worldwar3 \#wwiii \#ThatsWhatlLike \#foryou \#fyp \#4u \#viral \#foryoupage \\
\#notforyou \#nfyp
\end{tabular}

Hierin zeigen sich im Bezug auf den Militärdienst, eher stereotype bis «geschlechterkonforme» Ansichten zum «Krieg». So hoffen Frauen* und queere Personen, aufgrund ihres Geschlechts oder vermeintlicher sexueller Orientierung von Kriegsaktivitäten verschont zu bleiben. Auch wird das Rollenverständnis durch die Vertextlichung einer klassischen Abschiedsszene, in der der Mann die Frau zum Kriegsdienst verlässt, veranschaulicht:

just leave the gays at home. we can cook and clean your houses. \#ww3
\#fyp

Kiss your girl goodbye fellas \#ThatsWhatlLike \#fyp \#iran \#ww3 \#usa

\section{(B2) Vorbereitung auf die eigene Rekrutierung}

Auffallend sind zudem Caption-Inhalte, die den (patriotischen) Dienst für das Land thematisieren und die Rekrutierung zum Militär als gesellschaftliche Aufgabe und Pflicht benennen:

i would serve but i'm too old $\because \&$ \#morph \#ww3 \#fyp \#gymrush
\#turnitup \#HMmerchformation \#worldwar3
we are here to SERVE our country and SERVE these looks mama
We recruits and drafties need win ww3 lets get these blue coins boys
\#lego \#legostarwars \#fyp \#fypage \#fy \#jumanjichallenge \#aussie \#ww3
\#hot \#it


Proud to serve my country. \#pov \#war \#wwiii \#ww3 \#worldwar3 \#ceoof \#turnitup \#fyp \#foryoupage \#foryou \#comedy \#expectationvreality \#meme Smhh I ain't going to no in real life Call of Duty af ?fyp \#ww3 \#foru \#4u \#foryou

I'm glad Trump is looking to make peace with Iran but I was looking forward to being drafted and doing this with the boys \#kpop \#twice \#ww3

can't wait to get drafted \#ww3 \#drafted \#fyp \#worldwar3 \#resolutionunlocked

Darunter fällt auch das Vorbereiten für den Kriegsdienst und den Krieg: get ready soldiers. \#wwiii \#ww3 \#greenscreen well pack your bags boys it's go time; I hope all this call of duty paid off@ \#ww3 \#fyp \#xyzcba

see y'all at the battlefields my fellow boys \#fyp \#foryoupage \#wwiii \#worldwar3 \#trend \#viral \#trump \#2020 \#xyzcba \#ww3

\section{(B3) Der Moment der Rekrutierung}

Viele Captions verweisen auf eventuelle bzw. fiktive Zukunftsszenarien und zeigen einen thematisch starken Bezug zum Video, indem das Eingezogen werden als vergangenes \#ww3-Szenario «Realität» wurde:

When the national service come to the door \#foryou \#dhld \#ww3 \#viral

I've got my way out

Some draft scouts just showed up at my house to interview me..... \#wwiii \#lsw \#xyzbca \#fyp \#ThatsWhatlLike \#4u \#draft \#worldwar3 \#ww3

Got drafted 3 \#drafted \#goodbye2019hello2020 \#ww3 \#foryou

\#fypage \#foryourpage \#yougonedie @dickherdownmattt

Ive been drafted boys $\because$ \#fyp \#ww3 \#xyzbca

What are yalls numbers? I'm 071 and l'd be drafted :( \#greenscreen \#fyp \#ww3 \#wwiii \#foryoupage \#foryou \#draft \#ceoofchocolatemilk 
Dabei beinhalten die \#drafted-Captions in dieser Subkategorie auch thematischen Bezug zur TikTok-Community, indem das fiktive Kriegs-Szenario in der TikTokWelt gedacht wird:

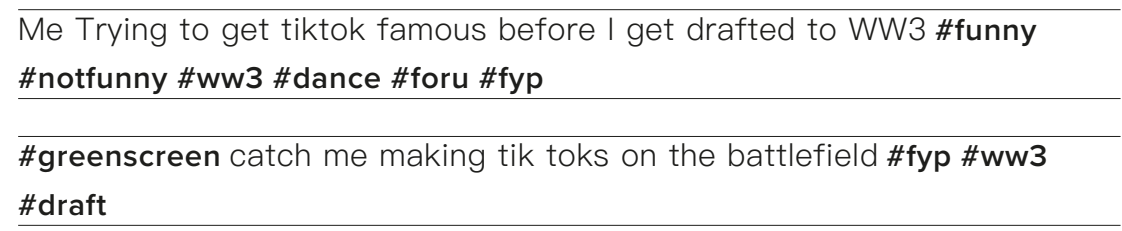

(B4) Verhinderung der eigenen Rekrutierung

Im Weiteren zeigen sich auch Gegenhaltung zum «bevorstehenden» Krieg oder Kriegsdienst, Verbalisierungen von Ängsten und eventuelle «Strategien» zum NichtEingezogen werden:

\begin{tabular}{l}
\hline ain't tryna join the war 0 \#WW3 \#fyp \#trend \#viral \#2020 \#4u \\
\#foryoupage \#foru \\
\hline I ain't going to no war \#greenscreen \#greenscreenvideo \#tiktoktraditions \\
\#ww3 \#fyp \#foryou \#foryoupage \\
\hline and that's on not getting drafted \#drafted \#ww3 \#tiktokww3 \#women \\
\#foryou \#foryoupage \#fyp \#jokes \\
\hline me talking to the drafting officer explaining why i can't participate in \\
world war 3 \#fyp \#ww3 \\
\hline Please dont let Donald take me \#foryourpage \#foru \#bigtimetok \\
\#wintervibes \#ww3 \\
I ain't going to no war \#fyp \#foryou \#foryourpage \#foryoupage \#ww3 \\
How to get out of the draft \#fy \#fyp \#foryou \#foryoupage \#ww3 \\
\#gymrush \#turnitup \#comedy \#funny \#viral \#trend \\
\hline POV: You're the \#worldwarthree recruiter and l'm avoiding draft \#pov \\
\#duetme \#duetthis \#ww3 \#wwiii \#funny \#wwiimemes \#fyp \#foryou \#gay \\
\#lgbt \#bi
\end{tabular}

Grundsätzlich wird die Information in den Captions in dem vorliegenden Datenset kontextualisiert und dekontextualisiert zum Inhalt des Videos dargestellt. Captions beinhalten meist konkrete Ansprachen an die Community wie Fragen, Statements und Szenarien. Fragen beziehen sich dekontextualisiert zu Video-Inhalten meist auf 
die Kommunikation mit anderen Nutzenden. Statements und Beschreibungen der Szenerien finden meist kontextualisiert zu Inhalten der Videos statt.

\subsection{Qualitative Analyse der Videos}

Im Sample befinden sich 953 distinkte Sounds, die teilweise mehrfach verwendet wurden. Dabei finden sich in der Gestaltung der Videos je nach Sound spezifische Muster. Für die qualitative Videoanalyse wurden zwei Sounds ausgewählt, die mehrfach verwendet wurden und sich jeweils einem der beiden oben genannten Themen zuordnen lassen.

\begin{tabular}{|l|l|l|}
\hline Soundname & $\begin{array}{l}\text { Videos dazu } \\
\text { im Sample }\end{array}$ & $\begin{array}{l}\text { Videos zum Sound insge- } \\
\text { samt (Stand Mai 2020) }\end{array}$ \\
\hline $\begin{array}{l}\text { «Everybody's Going Through Changes» } \\
\text { - Maya Rudolph, Jordan Peele und Mark } \\
\text { Rivers }\end{array}$ & 10 & 1667 \\
\hline «ww3 be like»- issywaha & & 32.400 \\
\hline
\end{tabular}

Tab. 1.: Soundname, Videos im Sample und Videos zum Sound insgesamt (\#ww3).

Es handelt sich dabei zum einen um einen Ausschnitt aus dem Song «Everybody's Going Through Changes» (VB) von Maya Rudolph, Jordan Peele und Mark Rivers, der neben einem Instrumentalteil die gesungene Textzeile «He's going through changes» umfasst und zu dem Videos zur Thematik des Eingezogen werdens erstellt werden. In diesen wird teilweise das komplette 15-sekündige Sample verwendet oder eine auf 10 Sekunden gekürzte Version, die den gesungenen Text abrupt nach «He's going through changes» abbrechen lässt.

Zum anderen handelt es sich um ein 14-sekündiges Medley der beiden Songs «Ain't your Mama» von Jenifer Lopez und «Hey Mama» von Nicki Minaj (VA), das von der Nutzerin @issywaha am 3. Januar auf die Plattform geladen und mit dem Titel "ww3 be like» versehen wurde. Im Original-Video wird \#ww3 im Rahmen von Textinsertierungen thematisiert, jedoch findet sich in der Caption kein Verweis und auch kein entsprechendes Hashtag, wodurch es nicht im vorliegenden Sample auftaucht. Es stellt ein Beispiel für einen Sound dar, der gestaltet wurde, um einen möglichen Kausalzusammenhang zwischen dem Ausbruch eines dritten Weltkriegs und den Veränderungen von Geschlechterrollen - hin zu stereotypen Rollenbildern - zu verweisen. So finden sich im ersten Teil des Sounds die Textzeilen «l ain't gon be cooking all day, I ain't your mama. I ain't gon' do your laundry, I ain't your mama», während es im zweiten Teil heisst: «Yes, I do the cooking. Yes, I do the cleaning».

7 https://www.tiktok.com/music/Everybodyss-Going-Through-Changes-6743043379182962689 (08.05.2020).

8 https://www.tiktok.com/music/ww3-be-like-6777807262015605509 (08.05.2020). 
Beide Sounds beinhalten dabei schon auf auditiver Ebene ein transformatorisches Moment, welches über die Textzeilen realisiert wird und von den Nutzenden auch in der Gestaltung der visuellen Ebene umgesetzt wird. So beschreiben sowohl die Videos zu VB einen Wandel des (meist männlichen) Akteurs als auch die Videos in VA Veränderungen in den (meist weiblichen) Akteurinnen. Dabei resultieren die beiden Sounds in einer unterschiedlichen Video-Ästhetik: Während in VB alle Videos (bis auf eines) mit Fotos arbeiten, die meist mithilfe des sogenannten facemorphingFilters ineinander übergehen, agieren in den Videos zu VA alle Personen vor der Kamera und montieren zu den einzelnen Textzeilen kurze schauspielerische Szenen aneinander. In allen Videos zu beiden Sounds wird mit Textinsertierungen gearbeitet, die auf eine "Transformation» hinweisen. Dabei fällt auf, dass die Videos zu VA durch die häufigste Insertierung "girls before ww3» und "girls after/during ww3» auf eine generelle Veränderung im Bereich geschlechtstypischen Handelns verweisen. Im Gegensatz dazu nutzen die VB Videos die Insertierungen um einen Ausgangspunkt für eine persönliche Transformation festzulegen. Sie starten durchgängig mit der Insertierung eines generalisierenden «me» oder «you», dem unterschiedliche Mutmassungen über Auswahlkriterien in Zusammenhang mit dem «draft» (engl. Eingezogen werden) begegnen (found out, hear(ing) etc.), die die Person motivieren sich zu verändern. Die Videos thematisieren dabei eine Vielzahl unterschiedlicher Ideen zur Begründung des Eingezogen werdens: Drei gehen davon aus, dass ausschliesslich Männer eingezogen werden können (VB_01, VB_02, VB_03), andere Videos vermuten, dass nur (bzw. verstärkt) junge (VB_05), heterosexuelle (VB_09), schlanke (VB_07) oder «weisse Menschen» (VB_10) eingezogen werden können. Ebenso wird vermutet, dass Menschen mit einer körperlichen Beeinträchtigung (VB_08) und schwangere Frauen (VB_04) nicht eingezogen werden könnten. Im Rahmen der Fotomontagen durchlaufen die Akteurinnen und Akteure jeweils eine Transformation, durch welche, die von ihnen vermutete Einzugs-Bedingung, die sie zu Beginn des Videos erfüllen, im letzten Bild nicht länger auf sie zutrifft. Eine Ausnahme stellt VB_06 dar, welches sich mit der Reaktion von Frauen generell auf die Information darüber auseinandersetzt, dass auch weibliche Personen eingezogen werden können und eine Flucht in tradierte Rollenklischees visuell umsetzt, wie sie auch in den VA-Videos zu sehen ist.

Damit entspricht VB_06 dem Hauptnarrativ derVA-Videos, welche sich wesentlich homogener darstellen als die zu VB erstellten Videos. Dies ist insbesondere durch den vorhandenen Text im Sound bedingt, welcher konkrete haushaltsbezogene Handlungen anspricht (cooking, laundry, cleaning) und mit einer Negativ- bzw. Positivwertung versieht, welche im Video «bespielt» werden. So starten alle Videos damit, eine weibliche Person (Ausnahme VA_01 zeigt zwei junge Frauen) in der Küche zu zeigen, die sich mit Mimik und Gestik von der Handlung des Kochens abgrenzt und diese in dem Moment dezidiert beendet, wenn die Sound-Stimme schlussfolgert «l ain't your mama». Im Anschluss präsentieren alle Videos eine Aufnahme in der Wäschekammer 
bzw. an der Waschmaschine, in der erneut die Handlung explizit abgebrochen wird, wenn die Schlussfolgerung «I ain't your mama» ertönt. Daraufhin ergänzen einige Videos eine dritte Szene für die Sequenz, in der ein weiteres Mal «l ain't your mama» ertönt, bei der ein Wischmop (VA_03) oder ein Staubsauger (VA_04) fallen gelassen werden, um sich von einer weiteren stereotypen Handlung abzugrenzen oder ostentativ die Schlussfolgerung in die Kamera gelipsynced wird (VA_01, VA_07), um ihre Aussage noch zu bekräftigen. Im zweiten Part des Medleys überstilisieren alle Akteurinnen eine positive Bewertung der Hausarbeiten, in einigen Videos durch die Entgegennahme eines entsprechenden Befehls (VA_04, VA_09) eingeleitet, welcher sich im Lipsyncing zu «Yes, I do the cooking» ablesen lässt. Der Bezug zu \#ww3 wird lediglich über die Textinsertierungen deutlich. Zwei der Videos nutzen den Sound und das entsprechende Hashtag jedoch auch, um nicht ww3-spezifische Einflüsse auf die Veränderung geschlechtstypischen Handelns zu thematisieren, so etwa vor und nach der Mutterschaft (VA_08) oder vor und nach dem Besuch von Sozialarbeitenden, die über das Kindswohl entscheiden sollen (VA_07). Dass der Sound unabhängig von der ww3-Thematik funktioniert, spiegelt sich auch in dem sehr hohen bisherigen VideoVorkommen wider, welches weit über die Dauer des \#ww3-Trends hinausgeht.

Anders als in den Videos zu VB, die - mit Ausnahme von VB_06 - erst im letzten Bild die Verwandlung des Protagonisten präsentieren, verwenden die Videos zu VA mehr Zeit auf die Darstellung des Transformationsergebnisses (ca. 4 Sekunden). Gemeinsam ist allen analysierten Videos, dass sie, trotz der Kürze der ihnen zur Verfügung stehenden Zeit, ein Narrativ gestalten, das von einer Ausgangssituation in eine davon verschiedene bzw. zu dieser konträren Ergebnissituation führt. Entscheidenden Beitrag zum Verständnis der Story leisten dabei die Text-Insertierungen, die ermöglichen, dass ein und dieselben Aufnahmen potenziell unterschiedliche Storys erzählen können. Damit ähneln sie Gestaltungsverfahren visueller Memes.

\section{Diskussion der Ergebnisse und Fazit}

Die Kombination aus quantitativer und qualitativer Analyse der einzelnen auf TikTok vorhandenen kommunikativen Ebenen zeigt, inwiefern die Mikro-Video-Plattform genutzt wird, um eigene Interpretationen von und Vermutungen über politische Informationen kreativ zu verarbeiten und miteinander zu teilen. Wenngleich der Wunsch nach Sichtbarkeit und Viralität eindeutig als wichtigstes Moment der TikTokKommunikation identifiziert werden konnte und in $86,6 \%$ der Fälle mit dem konkreten Thema verbunden wurde, zeigt die Hashtag-Analyse, dass der politikbezogene Trend \#ww3 zusätzlich verwendet wird, um solche Themen zu verhandeln, die die Nutzenden dazu in Beziehung setzen (31,6\% der Fälle). Dabei fiel auf, dass Nutzende einen Schwerpunkt darauf legen, eine präferierte Lesart für ihre Videos mitzugeben (32\% der Fälle). 
Auch die Caption-Analyse entlang der Themen «Gender» und «Eingezogen werden» zeigte, dass die Inhalte - bezogen auf \#ww3 - häufig durch die Vergabe weiterer Hashtags, die weniger thematisch, sondern eher plattformbezogen sind, ergänzt werden, um eine mögliche Viralität bzw. einen Trend zu erzeugen oder diesem zu folgen. Dies stellten auch Omar und Dequan (2020) fest, die im Produzieren von Inhalten mit dem Ziel der Viralität ein besonders hervortretendes Merkmal von TikTokNutzenden ausmachen. Inhaltlich präsentieren die hier analysierten Captions häufig überspitzte und damit mitunter irritierende Sichtweisen auf einen vermeintlich bevorstehenden Krieg. Sie zeigen vielfältige Formen der (politischen) Meinungsäusserung, die häufig mit der Verwendung stilistischer Mittel der Übertreibung verknüpft sind und auf diesem Wege Humor erzeugen. Die humorvolle und ironische Vermittlung von Inhalten auf TikTok unterstützt auch die Untersuchung von Wang (2020), die hieraus die kreativen Potenziale der Nutzenden erkennt. Dabei zeigt sich in den Inhalten der \#ww3-Captions auch die Einnahme vermeintlich provokanter Positionen, die auch im Zusammenhang mit Performativität und Selbstdarstellung gelesen werden können. Wie in der Studie von Khattab (2020) zeigt sich innerhalb der Captions und Videos eine meist heteronormative Darstellung von Geschlecht sowie der Verteilung von Aufgaben und «Care-Arbeit» innerhalb klassischer Rollenbilder, die jedoch mehrheitlich von einer ironischen Überhöhung durchzogen ist. Anders als in der Studie von Khattab (2020), konnten in den Videos und Captions zu \#ww3 keine sexualisierten Inhalte und konkreten Aussagen zu Körperbild und Schönheit identifiziert werden. Vielmehr zeigte sich eine deutliche Thematisierung von Männlichkeit und Weiblichkeit in stereotypen, heteronormativen Rollenbildern, die vermeintlich während eines Krieges und danach (re-)produziert würden. In der Überspitzung möglicher Szenarien liegt eine neue Form des Ausdrucks und der Verhandlung politisch und gesellschaftlich brisanter Inhalte in sozialen Medien - dies wurde von Literat und Kligler-Vilenchik (2019) herausgearbeitet und kann auch im Hinblick auf die oben genannten thematischen Bezüge von \#ww3 festgestellt werden. Ebenfalls lässt sich, ähnlich wie bei Literat und Kligler-Vilenchik (2019), feststellen, dass innerhalb der Captions Text und Hashtags als Codes dafür verwendet werden, welche politischen Positionen vertreten werden bzw. von welchen Meinungen sich abgegrenzt wird. Die vorliegende Studie kann die Ergebnisse von sowohl Literat und Kligler-Vilenchik (2019) als auch von Serrano et al. (2020) unterstützen, indem TikTok als ein Ort politischer Meinungsäusserung identifiziert werden kann. Darüber hinaus ist die Plattform als ein Raum für die Distribution, Kommunikation und Aneignung politischer Information bzw. Inhalte zu benennen, in dem experimentell künstlerische Prozesse der Nutzenden bei der Verhandlung und Verarbeitung politischer Information zum Tragen kommen. 
Im Vergleich mit der Video-Analyse fällt auf, dass das dort identifizierte Prinzip des Plot-Twists in den Captions nicht gespiegelt wird. Während die Videos somit zwei Perspektiven und/oder eine Reaktion auf eine Ausgangssituation präsentieren, thematisieren die Captions - auch aufgrund der Zeichenlimitierung - verstärkt eines der konkurrierenden Narrative im Video. Als prägendes Stilmittel in Schriftsprache und darstellendem Spiel konnte gleichermassen eine Überbetonung von Gefühlen durch Mimik, Emojis und Hashtags identifiziert werden, welche auf eine ironische Überhöhung und - damit verbunden - auf eine kritische Distanz zu den verhandelten Inhalten hindeutet. Dies resultiert darin, dass jeglicher Positionierung unmittelbar ein Moment der Unsicherheit innewohnt: Indem die Kommunikation beständig in der Schwebe gehalten wird - zwischen Realität und Fiktion, eigener Position und Rolle, konkurrierenden Narrativen etc. - gestalten die Nutzenden einen Schutzrahmen für sich und ihre Äusserungen. Dieser wird noch dadurch gestärkt, dass sie in Form von Hashtags dominante Lesarten für ihre Videos mitteilen und in der zugehörigen Kategorie «meaning \& reception» besonders häufig Lesarten aus dem Bereich Humor vorschlagen (etwa \#comedy, \#funny etc.). Wie in der qualitativen Videoanalyse gezeigt wurde, spielen gerade in Bezug auf die Auflösung des in der Einleitung erwähnten polysemischen Potenzials der audiovisuellen Aufnahmen Text-Insertierungen eine wichtige Rolle. Diese haben damit zugleich das Potenzial, eine tiefere Kommunikationsebene zu eröffnen, in welcher Inhalte parallel zu und unabhängig von den Bestrebungen nach Viralität, Sicherheit und Ästhetik verhandelt werden können. Es ist daher festzuhalten, dass eine Analyse der Aneignung politischer Information auf TikTok immer das vollständige Kommunikat mit all seinen Ebenen (Video, Caption und Hashtags) zu berücksichtigen hat und insbesondere die (inhaltlichen) Brüche und Verschiebungen zwischen den textuellen Elementen im Metatext und denen im Video selbst in den Fokus rücken sollte, um eine umfassende Analyse durchführen zu können. Hieraus ergibt sich für die medienpädagogische Praxis die Notwendigkeit einer ganzheitlichen Betrachtung der Inhalte und Aktivitäten Jugendlicher auf der Plattform, welche sich nicht auf die blosse Wahrnehmung des Bewegtbilds beschränken darf, sondern Herstellungsprozesse sowie die unterschiedlichen Ebenen und Strategien zur (gemeinschaftlichen) Bedeutungsproduktion mitberücksichtigen muss. In zukünftiger Forschung könnten phänomen- oder eventbasiert und im Vergleich bzw. in der Abgrenzung zu anderen Social Media Plattformen Alleinstellungsmerkmale in der Diffusion, Kommunikation und Aneignung politischer Information auf TikTok identifiziert und dadurch tiefere Kenntnisse über die Nutzung der Plattform im Zusammenhang mit politischer Information getroffen werden. Eine explorativ-qualitative Interviewstudie mit Nutzenden der Plattform würde zudem tiefere Einblicke in Motivation, Gefühle und Ziele während der Interaktion zulassen. 


\section{Limitationen}

Die Wahl des Erhebungsinstruments birgt das Risiko der systematischen Verzerrung, weshalb Einschränkungen aufgrund möglicher Verzerrungen und Folgerungen im Zuge der Analyse miteinbezogen werden müssen. Analysen sozialer Netzwerke bieten die Möglichkeit, über kurze Zeiträume in grossem Umfang soziale Interaktionen zu untersuchen. Dies kann helfen, einen grundlegenden Einblick in verschiedene Social Media Plattformen zu erhalten und Online-Phänomene, Beziehungen und Verhalten von Nutzenden zu untersuchen. Datenerhebungen im Rahmen von Social Media Analysen können meist kein strategisches Vorgehen zur Stichprobenziehung erfüllen, ermöglichen aber die Identifikation von Trends und Diskursen, was für die hier vorliegende Studie als zentral angesehen wird.

Bei der Durchführung der Studie stiessen wir auf ethische Fragen, die als Limitationen berücksichtigt werden sollten. Im Rahmen der Datenerhebung wurde die Plattform TikTok explizit nach öffentlichen Daten durchsucht und Inhalte wurden ohne das Wissen der Nutzenden gespeichert. Ein grosser Teil der Daten lässt auf minderjährige Nutzende schliessen. Angesichts des Alters der Jugendlichen, kann nicht ausgeschlossen werden, dass sich diese nicht über Konsequenzen bewusst sind, die sich aus der Verbreitung öffentlicher Inhalte ergeben. Um den Datenschutz einzuhalten, wurden in dieser Studie keine Bildelemente der Videos eingebunden, auch wurden Captions nicht in Zusammenhang mit Namen der Userinnen und User, Ort, Zeit und/ oder Datum der Posts veröffentlicht. Inhalte der Videos wurden ausgewertet und generalisiert zu Themen geclustert, um keine Rückschlüsse auf Nutzende zuzulassen. Nach der Analyse des empirischen Materials wurden nur die Daten sicher auf verschlüsselter Festplatte gespeichert, die notwendig sind, um die Reproduzierbarkeit der Studie zu ermöglichen. Das Vorgehen beim Scrapen von offenen Daten kann als Limitation der Studie angesehen werden, da Probandinnen und Probanden nicht aktiv am Forschungsprozess beteiligt sind bzw. diesen genehmigt haben. Dieses Vorgehen sollte daher nie ohne forschungsethische Reflexion erfolgen.

Darüber hinaus wurde entschieden, Vermutungen zu Ursachen eines möglichen bevorstehenden \#ww3 nicht in die Analyse miteinzubeziehen, um einer möglichen Verzerrung durch eine unilaterale Darlegung des Konfliktes vorzubeugen. Da Jugendliche z.B. in der islamischen Republik Iran keinen Zugang zu Social Media Apps wie TikTok haben, wäre damit nur eine verzerrte Sichtweise auf den Konflikt zwischen den Vereinigten Staaten und Iran zu erwarten. Der Diskurs zu \#ww3 könnte so vorrangig US-amerikanische und westeuropäische Perpektiven spiegeln. 


\section{Literatur}

Beutell, Jacqueline M. 2020. «Children's rights and social media: An analysis of TikTok's Terms of Service through the lens of a young user». In Proceedings from the 2019 University of Illinois Global Education Symposium. College of Education. University of Illinois at UrbanaChampaign: Office of International Programs. University of Illinois at Urbana-Champaign. http://hdl.handle.net/2142/106069.

Bo, Xiang. 2018. «Chinese video sharing app boasts $500 \mathrm{mln}$ monthly active users». Xinhua, 17. Juli 2018. http://www.xinhuanet.com/english/2018-07/17/c_137330644.htm.

Dawkins, Richard. 1976. The Selfish Gene. New York: Oxford University Press.

Dettwiler, Gabriela, und Reto Stauffacher. 2020. «Eine 18-Jährige beschwört auf Tiktok die Eskalation zwischen den USA und Iran zum ‘dritten Weltkrieg) herauf - und erreicht damit ein Millionenpublikum». Neue Zürcher Zeitung, 7. Januar 2020. https://www.nzz. ch/international/tiktok-der-3-weltkrieg-wird-zum-trend-und-sorgt-fuer-empoerungld.1532336? reduced=true.

Feld, Olivia. 2019. «TikTok Fined \$5.7m for Violating Children's Privacy». The Telegraph, 28. Februar 2019. https://www.telegraph.co.uk/technology/2019/02/28/tiktok-fined-57m-violating-childrens-privacy/.

Herrman, John. 2019. «How TikTok Is Rewriting the World». The New York Times, 10. März 2019, Abschn. Style. https://www.nytimes.com/2019/03/10/style/what-is-tik-tok.html.

Höfer, Fritz. 2019. «TokTok - App-Musicking als aktuelle jugendkulturelle Musikpraxis in ihrer Relevanz für die Musikpädagogik.» Herausgegeben von Gabriele Enser, Bernhard Gritsch, und Fritz Höfer. Musikalische Sozialisation und Lernwelten. Münster: Waxmann.

Khattab, Mona. 2020. «Synching and Performing: Body (Re)-Presentation in the Short Video App TikTok». Widerscreen.fi 1-2. http://widerscreen.fi/numerot/2019-1-2/synching-andperforming-body-re-presentation-in-the-short-video-app-tiktok/.

Knuth, Hannah. 2019. «TikTok: Die Unschuld verloren». Die Zeit, 6. Dezember 2019, Abschn. Digital. https://www.zeit.de/2019/51/tiktok-zensur-china-social-media-internet.

Köver, Chris. 2020. «Diskriminierende Moderationspraktiken. Zu hässlich für TikTok». Netzpolitik.org, 17. März 2020. https://netzpolitik.org/2020/zu-haesslich-fuer-tiktok/.

Krippendorff, Klaus. 2019. Content Analysis: An Introduction to Its Methodology. Fourth edition. Los Angeles: SAGE.

Laufer, Daniel, und Chris Köver. 2020. «NetzDG-Bericht. TikTok löscht und sperrt am häufigsten politische Inhalte». Netzpolitik.org, 31. Januar 2020. https://netzpolitik.org/2020/tiktokloescht-und-sperrt-am-haeufigsten-politische-inhalte/.

Literat, loana, und Neta Kligler-Vilenchik. 2019. «Youth Collective Political Expression on Social Media: The Role of Affordances and Memetic Dimensions for Voicing Political Views». New Media \& Society 21 (9): 1988-2009. https://doi.org/10.1177/1461444819837571. 
Medina Serrano, Juan Carlos, Orestis Papakyriakopoulos, und Simon Hegelich. 2020. «Dancing to the Partisan Beat: A First Analysis of Political Communication on TikTok». In 12th ACM Conference on Web Science, 257-66. Southampton United Kingdom: ACM. https://doi. org/10.1145/3394231.3397916.

Meyer, Thomas. 2001. Mediokratie: die Kolonisierung der Politik durch das Mediensystem. Bd. 2204. Edition Suhrkamp. Frankfurt am Main: Suhrkamp.

Müller, Antje. 2019. «Stichwort Tick Tack, Tik Tok». merz | medien + erziehung 3 (Digitalität. Religion. Pluralismus). https://www.merz-zeitschrift.de/alle-ausgaben/pdf/antjemllerstichwortticktacktiktok/.

Müller, Valérie. 2020. «User*innen verarbeiten ihre Angst vor einem dritten Weltkrieg in Memes». Süddeutsche Zeitung, 6. Januar 2020, Abschn. jetzt.de. https://www.jetzt.de/politik/ twitter-und-tiktok-trend-wwiii-user-verarbeiten-ihre-angst-vor-einem-dritten-weltkriegin-memes.

Omar, Bahiyah, und Wang Dequan. 2020. «Watch, Share or Create: The Influence of Personality Traits and User Motivation on TikTok Mobile Video Usage». International Journal of Interactive Mobile Technologies (iJIM) 14 (04): 121. https://doi.org/10.3991/ijim.v14i04.12429.

Petter, Jan. 2020. «Wir haben mit der Influencerin gesprochen, die auf TikTok vor einem Weltkrieg warnte - und jetzt angegriffen wird». DER SPIEGEL (online), 9. Januar 2020, Abschn. bento.de. https://www.spiegel.de/panorama/iran-konflikt-tiktok-influencerin-warnt-vordrittem-weltkrieg-jetzt-wird-sie-angegriffen-a-b7899a39-4830-4659-92a5-a9bf794271f1.

Rezo. 2020. «Internet-Memes: Es ist zu ernst, um ernst zu bleiben». Die Zeit, 14. Januar 2020, Abschn. Kultur. https://www.zeit.de/kultur/2020-01/internet-memes-weltkrieg-witze-iranusa-rezo.

Romano, Aja. 2020. «Coping with war and crisis through memes. Do what you need to cope with World War III: Memes are the new therapy». Vox, Vox Media, 17. Januar 2020. https://www. vox.com/2020/1/17/21065113/world-war-3-memes-iran-2020-saleem-alhabash-interview.

Röser, Jutta, und Corinna Peil. 2010. «Räumliche Arrangements zwischen Fragmentierung und Gemeinschaft: Internetnutzung im häuslichen Alltag». In Alltag in den Medien - Medien im Alltag, herausgegeben von Jutta Röser, Tanja Thomas, und Corinna Peil, 220-41. Wiesbaden: VS Verlag für Sozialwissenschaften. https://doi.org/10.1007/978-3-531-91949-2_13.

Schulz, Winfried. 2011. Politische Kommunikation. Theoretische Ansätze und Ergebnisse empirischer Forschung. Wiesbaden: VS Verlag für Sozialwissenschaften. https://doi. org/10.1007/978-3-531-93094-7.

Shifman, Limor. 2014. Memes in Digital Culture. The MIT Press Essential Knowledge Series. Cambridge Massachusetts, London England: The MIT Press.

Smolentceva, Natalia. 2019. «TikTok: World's most successful video app faces security concerns». Deutsche Welle, 27. März 2019, Abschn. Business. https://p.dw.com/p/3Ffbh.

Steadman, Otillia, und Ryan Brooks. 2020. «The Threat Of World War III Has Become A Meme And No One Knows How To Feel About It». BuzzFeed News, 3. Januar 2020. https://www. buzzfeednews.com/article/otilliasteadman/world-war-memes-tiktok-trump-iran-draftjokes. 
Wagner, Ulrike, und Christa Gebel. 2014. Jugendliche und die Aneignung politischer Information in Online-Medien. Wiesbaden: Springer Fachmedien Wiesbaden. https://doi. org/10.1007/978-3-658-04201-1.

Wang, Yunwen. 2020. «Humor and Camera View on Mobile Short-Form Video Apps Influence User Experience and Technology-Adoption Intent, an Example of TikTok (DouYin)». Computers in Human Behavior 110 (September): 106373. https://doi.org/10.1016/j.chb.2020.106373.

Weber, Amelie Marie. 2020. «TikTok-App. Das sagt der Chef zum Umgang mit Polit-Inhalten. Ein Video auf TikTok sorgt für Angst vor dem Dritten Weltkrieg». Berliner Morgenpost, 17. Januar 2020. https://www.morgenpost.de/politik/article228132853/TikTok-Kinderschuetzerwarnen-Eltern-vor-Gefahr-und-haben-Forderung.html.

Weichert, Rune. 2020. «Dritter Weltkrieg): Influencerin erklärt den Iran-USA-Konflikt und wird mit Kritik überhäuft». Stern online, 8. Januar 2020. https://www.stern.de/digital/influencerin-erklaert-dritten-weltkrieg-in-tiktok-video---und-es-hagelt-kritik-9077606.html.

Winkler, Sabine. 2019. «Tiktok ist die beliebteste App der Welt». DIE WELT, 6. Juni 2019. https:// www.welt.de/kmpkt/article194801553/Tiktok-ist-die-beliebteste-App-der-Welt.html.

Yeh, Oliver. 2019. «TikTok Surpasses One Billion Installs on the App Store and Google Play». SensorTower, 26. Februar 2019. https://sensortower.com/blog/tiktok-downloads-one-billion.

Zolfagharifard, Ellie. 2019. «The Incredible Rise of TikTok in the West Raises Questions for Regulators». The Telegraph, 3. April 2019. https://www.telegraph.co.uk/technology/2019/04/03/ incredible-rise-tiktok-west-raises-questions-regulators/.

Zuo, Hui, und Tongyue Wang. 2019. «Analysis of Tik Tok User Behavior from the Perspective of Popular Culture». Frontiers in Art Research 1 (3): 5. https://doi.org/10.25236/FAR.20190301. 